\title{
CRITERI E PROBLEMI COSTRUTTIVI DI STRUMENTI PER MISURE DI RESISTIVITA' (*)
}

\section{Luigi Solaini}

1. - Tra i vari metodi geofisici per la esplorazione del sottosuolo, quello di resistività è uno dei più lungamente sperimentati e maggiormente conosciuti e sembra ormai cbe non resti molto da dire, né sulla costruzione degli strumenti di misura, né sulle modalità operative, né su quelle interpretative.

Però l'incremento della precisione che si desidera ottenere, comune a tutti i procedimenti geofisici, e la notevole profondità a cui si cerca di spingere la investigazione elettrica del terreno, pongono diversi problemi di carattere operativo, che ritengo utile riassumere brevemente.

Qualunque sia il procedimento pratico di misura, accade sempre cbe la differenza di potenziale tra gli elettrodi potenziometrici, che chiamerò in seguito brevemente sonde, decresce quando aumenta la distanza tra gli elettrodi di corrente e diviene molto piccola per lunghi profili, specialmente se il terreno è buon conduttore; il rapporto tra la differenza di potenziale $V$ misurata e la corrente $I$ inviata nel terreno scende allora a valori assai bassi. Se infatti ad esempio il sottosuolo ha una resistività media di $50 \Omega \mathrm{m}$, i valori del rapporto $\frac{V}{I}$ in funzione della distanza $d$ tra gli elettrodi di corrente è data dalla seguente tabellina, nelle due ipotesi che la distanza $a$ tra le sonde sia un terzo (metodo Wenner) o un decimo di $d$.

$$
\begin{array}{rrrrrrrrr}
d=2 & 4 & 6 & 8 & 10 & 20 & k m & \\
\frac{V}{I}=11.9 & 6.0 & 4.0 & 3.0 & 2.4 & 1.2 & 10^{-3} \Omega & \frac{a}{d}=\frac{1}{3} \\
\frac{V}{I}=3.2 & 1.6 & 1.1 & 0.8 & 0.6 & 0.3 & 10^{-3} \Omega & \frac{a}{d}=\frac{1}{10}
\end{array}
$$

Poiché la resistività media del terreno può esere anche inferiore

(*) Comunicazione presentata al Convegno dell'Associazione Geofisica Italiana, tenuto a Roma il 10-11 giugno 1952. 
a $50 \Omega \mathrm{m}$, ciò significa che uno strumento moderno deve essere capace di misurare il rapporto $\frac{V}{I}$ con approssimazione non inferiore a $10^{-5} \Omega$; se si determinano separatamente tensione e corrente, ammesso di inviare nel terreno una corrente di $1 A$, il voltmetro deve dunque avere una sensibilità di almeno 10 ॥ $V$. La misura della corrente non offre invece difficoltà.

Quando si giunge a sensibilità così elevate, diversi problemi si affacciano di soluzione tutt'altro che facile. Essi riguardano:

a) la scelta del tipo di corrente da inviare, che può essere continua o alternata o commutata; nel secondo caso la scelta della frequenza più opportuna;

b) il tipo più conveniente di stendimento;

c) la costruzione degli apparati di misura.

In questa breve nota desidero esporre alcune considerazioni relative ai tre punti considerati, perché penso che la loro discussione presenti una certa utilità.

2. - E ben noto che tanto la corrente continua quanto quella alternata hanno diversi inconvenienti.

Nei lunghi stendimenti, le differenze di potenziale dovute alle correnti telluriche, capricciosamente variabili, e i disturbi dovuti a correnti artificialmente immesse nel terreno, giungono a valori dello stesso ordine di grandezza di quelle che si devono misurare, per cui le misure in corrente continua divengono oltremodo penose se non impossibili.

Inoltre l'impiego degli elettrodi impolarizzabili non è del tutto scevro da inconvenienti, perché tra i due elettrodi esiste sempre una differenza di potenziale che raggiunge e supera $1 \mathrm{mV}$ e le cui variazioni possono influire sulle misure di precisione.

D'altra parte, anche operando in corrente alternata, se si eliminano completamente le influenze delle forze elettromotrici di contatto, non ci si può esimere dalla influenza delle correnti alternate circolanti nel terreno per effetto delle terre delle linee di trasporto della energia ecc. In recenti prove effettuate in Lombardia, in una zona tranquilla, lontana da linee elettriche, si è registrata con un oscillografo una differenza di potenziale alternata a $50 \mathrm{~Hz}$ di qualche centinaio di $u \mathrm{~V}$, tra due elettrodi posti a $300 \mathrm{~m}$ di distanza.

L'importanza di questi disturbi è legata alla frequenza della corrente che si impiega, la quale a sua volta dipende dalla profondità a cui si spinge la esplorazione e da difficoltà strumentali. Queste ultime 
fanno preferire la frequenza più elevata possibile, compatibile con la profondità di penetrazione; per profili lunghi alcuni chilometri, è senz'altro sufficiente una frequenza non inferiore a $20 \mathrm{~Hz}$, mentre quando si superano i $10 \mathrm{~km}$ (cosa che invero accade assai di rado, perché il potere risolutivo delle misure di resistività diviene assai piccolo) è necessario adottare frequenze di pochi Hertz.

Nel primo caso che, ripetiamo, è di gran lunga il più comune, la frequenza della corrente immessa non è molto diversa da quella della corrente industriale e quindi la eliminazione dei disturbi diviene un problema complesso dal punto di vista strumentale.

Finalmente, si potrebbe impiegare corrente commutata, ciò che del resto si fa ordinariamente con alcune apparecchiature, ma per gli errori che questo procedimento comporta, ritengo la soluzione inadatta a misure di precisione e a lunghi stendimenti, benché la corrente commutata abbia il vantaggio di eliminare gli inconvenienti della corrente continua senza dover cambiare gli strumenti, salvo la introduzione dei commutatori.

Concludendo, si può dire che nessun tipo di corrente è ideale per i lunghi sondaggi elettrici verticali; tuttavia ritengo che la corrente alternata a bassa frequenza sia quella più opportuna, perché la eliminazione dei disturbi può essere ottenuta in modo soddisfacente con opportuni dispositivi strumentali e con la scelta del tipo di quadripolo.

Parlando perciò degli strumenti di misura, ci limiteremo a considerare $\mathrm{i}$ problemi relativi all'impiego della corrente alternata.

3. - Esaminiamo ora brevemente le modalità operative, ma solo dal punto di vista della esecuzione delle misure e non da quello interpretativo.

Le questioni da considerare sono principalmente due e cioè la intensità della corrente da inviare nel terreno e la disposizione degli elettrodi.

La tendenza più recente relativa alla corrente, è quella di inviare forti intensità, anche di alcuni ampère, per mezzo di generatori di qualche chilowatt di potenza, in modo da ottenere anche negli stendimenti più lunghi e nei terreni meno resistivi, una differenza di potenziale sufficientemente elevata. Siccome le resistenze di contatto degli elettrodi e quelle del terreno nelle loro immediate vicinanze ammontano generalmente a diverse centinaia di ohm, per ottenere correnti di alcuni ampère, le tensioni del generatore devono avere va- 
lori elevati, che raggiungono anche $1000 \mathrm{~V}$. L'uso di tensioni cosi forti non è scevro di inconvenienti, sia perché richiede cavi molto bene isolati in tutta la loro lunghezza, sia perché può presentare dei pericoli per gli uomini addetti agli spostamenti degli elettrodi.

Si può pensare allora di diminuire la intensità di corrente aumentando in pari tempo la sensibilità degli strumenti, ma si urta contro una duplice difficoltà. La prima è di natura prettamente strumentale, perché, malgrado i grandi progressi realizzati nella costruzione di strumenti elettrici di misura e i miracoli della tecnica elettronica a cui si può attingere per studiare moderne apparecchiature di prospezione, non è facile e soprattutto non è opportuno superare in strumenti di campagna, certe sensibilità. Abbiamo visto che già con la corrente di un ampère, la sensibilità richiesta al voltmetro è di pochi microvolta; essa rappresenta a mio parere presso a poco la massima sensibilità utile in strumenti di campagna.

La seconda difficoltà è determinata dalla presenza delle correnti vaganti che sono talvolta tutt'altro che trascurabili, anche impiegando corrente alternata, malgrado gli eventuali accorgimenti strumentali atti a diminuire la loro influenza.

Quando aumenta la lunghezza dello stendimento, se la resistività apparente è costante, da un lato diminuiscono le differenze di potenziale misurate, dall'altro aumenta inevitabilmente la distanza tra le sonde e quindi l'influenza dei disturbi. Occorre quindi che la potenza del generatore sia sufficientemente grande da assicurare in ogni caso una differenza di potenziale maggiore di quella creata dalle correnti vaganti; tale potenza non può quindi scendere al disotto di $1 \mathrm{kw}$ per i più lunghi profili; se ci si limita a profili di 5 o $6 \mathrm{~km}$, può essere sufficiente una potenza di mezzo chilowatt. Naturalmente è più che opportuno, nei limiti del possibile, che la sensibilità strumentale sia notevole. Circa il tipo di stendimento, ritengo tra tutti preferibile quello adottato dalla Compagnie Générale de Géophysi đue e da qualche tempo costantemente impiegato anche dal mio Istituto.

Mantenere fissi in generale gli elettrodi offre, infatti, oltre a quello di una maggiore speditezza di lavoro, il vantaggio notevole che le misure sono ben poco influenzate dalle eterogeneità superficiali; alcuni sondaggi elettrici, effettuati tanto con lo stendimento Wenner, quanto con quello a sonde fisse, mostrano chiaramente la superiorità di quest'ultimo procedimento. Poiché mantenendo ferme le sonde varia da una misura all'altra il rapporto tra la loro distanza $a$ e quella $d$ tra 
gli elettrodi di corrente, si fa in modo che a sia tanto piccolo rispetto a $d$, da poter trascurare nelle formule interpretative il rapporto $\left(\frac{a}{d}\right)^{2}$. Le differenze di potenziale che si misurano sono al. lora nettamente inferiori a quelle ottenibili con lo stendimento di Wenner, ad esempio, e richiedono una elevata sensibilità dello strumento. E bene tuttavia far notare che l'aumento della distanza tra le sonde crea un beneficio illusorio riguardo alla precisione di misura, perché crescono contemporaneamente le differenze di potenziale dovute alle correnti vaganti.

A conclusione di queste brevi considerazioni, mi sembra si possa stabilire la necessità di usare voltmetri di sensibilità pari a pochi microvolta, correnti non eccessivamente elevate, ma tali da consentire misure corrette anche in presenza di disturbi e di adottare stendimenti con sonde fisse e ravvicinate.

4. - Passiamo finalmente in rassegna le diverse possibili soluzioni strumentali, considerandone i vantaggi e gli inconvenienti. è evidente anzitutto la necessità di non prelevare corrente dalle sonde; ciò può essere ottenuto o usando dei voltmetri elettronici, oppure mediante opposizione.

La prima soluzione, molto semplice e già attuata in alcuni strumenti, ha il grave difetto della incostanza della amplificazione del voltometro, il quale deve essere tarato ad ogni misura con una tensione campione. Le misure sono perciò piuttosto lente e la precisione raggiungibile non è molto elevata, specialmente trattandosi di strumenti di alta sensibilità e quindi dotati di amplificazione piuttosto forte. Inoltre è molto difficile la eliminazione dei disturbi, ciò che determina gli inconvenienti che abbiamo già esaminati.

La seconda soluzione, indubljiamente migliore, presenta tuttavia alcune difficoltà. La opposizione deve essere fatta con una tensione della identica frequenza della corrente inviata nel terreno e in fase con essa; bisogna perciò, o prelevare una frazione della corrente energizzante stessa, o disporre di un piccolo alternatore ausiliario calettato sullo stesso asse del generatore di corrente. Nel primo caso il prelevamento della corrente può essere ottenuto solo attraverso un trasformatore o con altri artifici, poiché altrimenti si porterebbe una sonda allo stesso potenziale di un elettrodo di corrente; data la notevole sensibilità degli strumenti di misura, è difficile tuttavia evitare con i 
trasformatori piccole fughe di natura elettrostatica, dannosissime perché non si può introdurre uno schermo elettrostatico efficiente. Sembra perciò più razionale l'uso del secondo generatore, montato con la cura necessaria per evitare sfasamenti tra le due correnti.

La misura può essere effettuata o con un unico strumento a bobine incrociate, seguendo lo schema adottato nel Megger geofisico, oppure con un amperometro ed un microvoltmetro indipendenti. Nel primo caso è molto difficile raggiungere una elevata sensibilità, perciò questa soluzione sembra da scartarsi senz'altro. Nel secondo caso, oltre all'amperometro, occorre un microvoltmetro di precisione, oppure delle resistenze non induttive tarate; quest'ultimo metodo è preferibile al

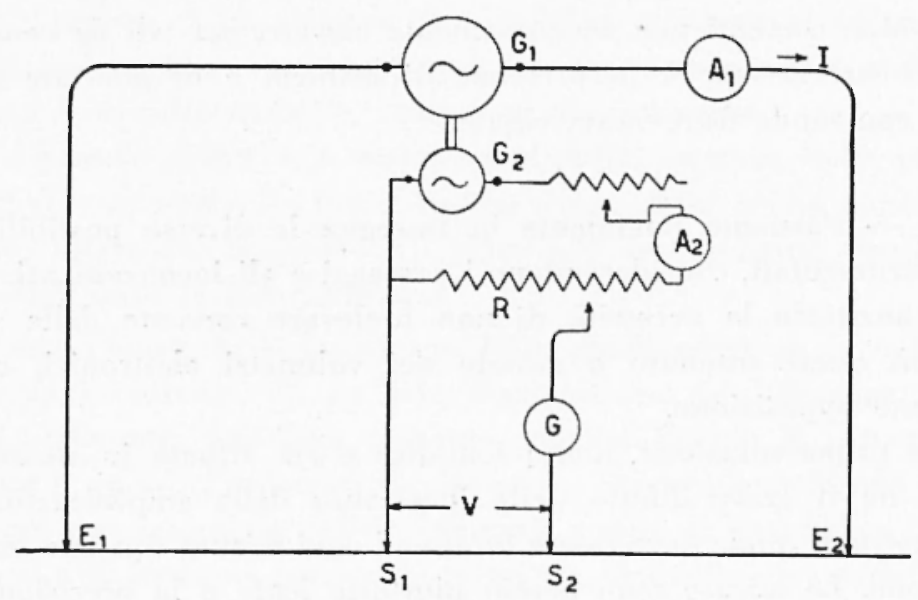

Fig. 1

primo, perché l'uso di uno strumento di misura a corrente alternata e di elevata sensibilità è particolarmente delicato. In entrambi i casi è necessario un galvanometro per stabilire l'equilibrio tra la tensione di opposizione e quella esistente tra le sonde; esso deve avere una sensibilità voltmetrica non inferiore a qualche diecina di microvolta per parte, cioè deve possedere praticamente la massima sensibilità ottenibile in strumenti portatili a corrente alternata. Il tipo di strumento che sembra più adatto è il galvanometro a vibrazione, tuttavia abbastanza ingombrante e molto costoso. Poiché questi strumenti sono molto sensibili a tensioni elettrostatiche, è opportuno ricorrere al generatore ausiliario per ottenere la corrente di opposizione; siccome tale corrente è indipendente da quella inviata nel terreno, occorre un 
apposito strumento che la misuri o che permetta di ridurla ad un valore costante.

Lo schema di un apparato disegnato secondo i concetti enunciati risulta perciò quello della fig. 1. Per quanto abbastanza complesso, esso rappresenta una delle migliori soluzioni per le misure di resistività in corrente alternata; del resto è molto simile ad alcuni strumenti a corrente continua comunemente in uso, salvo la introduzione del

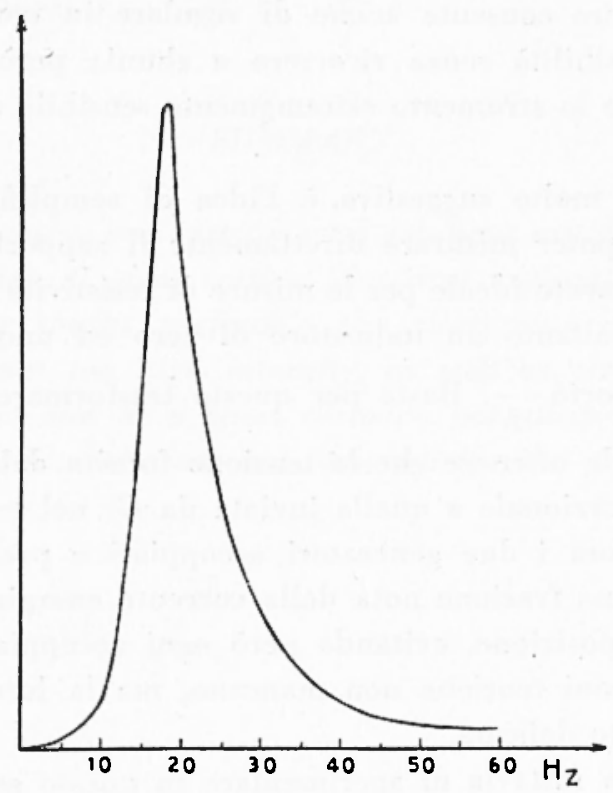

Fig. 2

generatore $G_{\Omega}$ direttamente accoppiato a quello principale $G_{1}$ al posto di una pila indipendente dalla batteria energizzante. E solo opportuno osservare che la resistenza $R$ è particolarmente delicata, perché deve variare da alcune centinaia di ohm a qualche decimo di ohm e deve essere letta con l'approssimazione di almeno $10^{-2} \Omega$; in pratica è necessario ricorrere ad un attenuatore.

La grande sensibilità necessaria per il galvanometro $G$ può essere diminuita anteponendogli un amplificatore; siccome lo strumento serve solo per stabilire l'equilibrio tra due tensioni opposte, la introduzione dell'amplificatore non determina inconvenienti sensibili, mentre ha il vantaggio di potere introdurre dei filtri atti a ridurre notevol- 
mente i disturbi che abbiano frequenza sensibilmente diversa da quella della corrente energizzante. Lo studio di tale amplificatore e del filtro non è molto semplice, a causa della bassa frequenza della corrente impiegata; tuttavia si può ottenere una curva di selettività come quella riprodotta in fig. 2 ; come si vede, se la sintonia si verifica per la frequenza di circa $20 \mathrm{~Hz}$, tutte le frequenze inferiori a $5 \mathrm{~Hz}$ sono praticamente tagliate, mentre le tensioni con le frequenze di $40 \mathrm{~Hz}$ sono già ridotte a $1 / 20$ del loro valore.

L'amplificatore consente anche di regolare in modo semplice e continuo la sensibilità senza ricorrere a shunt; però ha l'inconveniente di rendere lo strumento estremamente sensibile alle fughe elettrostatiche.

Finalmente, molto suggestiva è l'idea di semplificare l'uso dell'apparato e di poter misurare direttamente il rapporto tra tensione e corrente; l'apparato ideale per le misure di resistività sarebbe quello che possedesse soltanto un indicatore di zero ed uno strumento di misura del rapporto $\frac{V}{I}$. Basta per questo trasformare lo schema di fig. 1 , in modo da ottenere che la tensione fornita dal generatore $G_{2}$ sia sempre proporzionale a quella inviata da $G_{1}$ nel terreno. Bisogna abbandonare allora $i$ due generatori accoppiati e prelevare dal circuito primario una frazione nota della corrente energizzante, per utilizzarla per l'opposizione, evitando però ogni accoppiamento elettrostatico; le soluzioni teoriche non mancano, ma la loro realizzazione meccanica è molto delicata.

Vale la pena tuttavia di sperimentare in questo senso, perché si riuscirebbe ad ottenere così uno strumento di uso veramente pratico e di sensibilità molto elevata.

Un ultimo cenno sugli strumenti a registrazione fotografica; ̀̀ evidente che, volendo ricorrere al metodo di misura per opposizione, la registrazione può avvenire solo dopo l'azzeramento del galvanometro; l'oscillografo sostituisce semplicemente l'amperometro e il millivoltmetro. Il vantaggio della registrazione è perciò molto modesto, a meno che non si pensi alla possibilità di registrare anche la corrente passante per il galvanometro, ciò che consentirebbe di effettuare una opposizione approssimata e di correggere successivamente le indicazioni della registrazione voltmetrica.

Milano - Istituto di Geofisica appl. del Politecnico - Giugno 1952. 


\section{RIASSUNTO}

$\mathrm{Si}$ discutono le varie soluzioni operative e strumentali relative alla esecuzione di grandi sondaggi elettrici verticali. Si auspica la adozione di correnti alternate a bassa frequenza e di intensità non troppo elevata e di stendimenti eseguiti con sonde potenziometriche fisse e ravvicinate.

Si passano finalmente in rassegna le possibili soluzioni per gli strumenti di misura.

\section{SUMMARY}

Various operative and instrumental solutions are discussed concerning the execution of great vertical electrical explorations.

The Author thinks preferable the alternating currents at low frequency and not too high intensity, as well as electrode arrangements with fixed and at a short distance potentiometric electrodes.

Finally, possible solutions are reviewed for the potential measuring circuits. 